Portland State University

PDXScholar

$1-1-2004$

\title{
Low-Frequency Surface Wave Propagation and the Viscoelastic Behavior of Porcine Skin
}

\author{
Donald D. Duncan \\ Portland State University \\ Sean J. Kirkpatrick \\ Li Fang
}

Follow this and additional works at: https://pdxscholar.library.pdx.edu/ece_fac

Part of the Electrical and Computer Engineering Commons Let us know how access to this document benefits you.

\section{Citation Details}

Sean J. Kirkpatrick, Donald D. Duncan and Li Fang, "Low-frequency surface wave propagation and the viscoelastic behavior of porcine skin", J. Biomed. Opt. 9, 1311 (2004); http://dx.doi.org/10.1117/ 1.1803843

This Article is brought to you for free and open access. It has been accepted for inclusion in Electrical and Computer Engineering Faculty Publications and Presentations by an authorized administrator of PDXScholar. Please contact us if we can make this document more accessible: pdxscholar@pdx.edu. 


\section{Low-frequency surface wave propagation and the viscoelastic behavior of porcine skin}

\author{
Sean J. Kirkpatrick \\ Oregon Health and Science University \\ Department of Biomedical Engineering \\ Portland, Oregon \\ E-mail: sean.kirkpatrick@bme.ogi.edu
}

\section{Donald D. Duncan}

The Johns Hopkins University

Applied Physics Laboratory

Laurel, Maryland

\section{Li Fang}

Oregon Health and Science University Department of Biomedical Engineering Portland, Oregon

\begin{abstract}
A physical model describing the propagation of lowfrequency surface waves in relation to the viscoelastic behavior of porcine skin is presented, along with a series of empirical studies testing the performance of the model. The model assumes that the skin behaves as a semi-infinite, locally isotropic, viscoelastic halfspace. While the assumption of a semi-infinite body is violated, this violation does not appear to have a significant impact on the performance of the model based on the empirical studies. $1-\mathrm{Hz}$ surface waves in the skin propagate primarily as Rayleigh waves with a wavelength and velocity of approximately $3 \mathrm{~m}$ and $3.0 \mathrm{~m} / \mathrm{s}$, respectively. The amplitude of the acoustic wave, as measured by tracking the acoustic stress wave-induced shift in a backscattered laser speckle pattern, decreases exponentially with lateral distance from the acoustic source. Using this model of surface wave propagation, the mechanical loss factor or $\tan \delta$ of the skin is measured to be on the order of $0.14 \pm 0.07$. The results presented are consistent with earlier works on the propagation of low-frequency acoustic waves in biological tissues, and should serve as a theoretical and empirical basis for using the wave characteristics of propagating surface waves in combination with the mechanical behavior of the tissue for biomechanical studies and for potential diagnostic applications. ๑ 2004 Society of Photo-Optical Instrumentation Engineers. [DOI: 10.1117/1.1803843]
\end{abstract}

Keywords: low frequency; surface wave propagation; skin; acoustic wave.

Paper 03115 received Sep. 19, 2003; revised manuscript received Mar. 16, 2004; accepted for publication Mar. 19, 2004.

\section{Introduction}

The behavior of low-frequency surface waves in biological tissues has received only limited attention in the literature. Yet tracking low-frequency surface acoustic wave propagation in tissues provides a noninvasive means of investigating the mechanical behavior of the tissue. Such an approach may yield diagnostic methods based on local differences in mechanical behavior of tissues that may be indicative of certain pathologies. Indeed, this is the basis of manual palpation.

Potts, Chrisman, and Buras ${ }^{1}$ investigated the propagation and attenuation of low-frequency waves in human skin over a frequency range from near zero to $1 \mathrm{kHz}$. Their results indicated that at frequencies below a few hundred hertz, the waves propagated primarily along the skin surface. At higher frequencies, the waves tend to propagate as bulk waves. They inferred from these results that at low frequencies, the waves provide information from the surface layer (i.e., the stratum corneum), and at higher frequencies, information about the dermis is provided. Karzakov and Klochkov ${ }^{2}$ investigated the mechanical properties of skin tissues in the human arm over a range of 10 to $100 \mathrm{~Hz}$. One of their key results was that at these low frequencies, the speed of propagation was relatively slow, ranging between 21.0 and $1.6 \mathrm{~m} / \mathrm{s}$, respectively. They also noted that there is a very large interindividual variation in

Address all correspondence to Sean J. Kirkpatrick, Department of Biomedical Engineering, 20000 NW Walker Rd., Beaverton, OR 97006. Tel: 503-748-1320; E-mail: sean.kirkpatrick@bme.ogi.edu the speed of propagation at a given frequency. These results are consistent with those of Potts, Chrisman, and Buras. ${ }^{1}$

Low-frequency surface wave propagation has been measured in other tissues besides skin. Ganesan, Man, and Lai-Fook ${ }^{3}$ evaluated surface waves in lung tissue as a function of transpulmonary pressure as a means of evaluating lung edema. At frequencies $<10 \mathrm{~Hz}$, they observed propagation velocities between 2.87 and $1.8 \mathrm{~m} / \mathrm{s}$, depending on the transpulmonary pressure. Kirkpatrick, Hinds, and Duncan ${ }^{4}$ evaluated the propagation and decay of $1-\mathrm{Hz}$ acoustic waves in bovine nuchal elastin as a means of noninvasively determining the viscoelastic properties of this tissue for applications as a tissue engineering scaffold.

The purpose of this study was to evaluate low-frequency surface wave propagation and decay in porcine skin ex vivo and to directly relate the wave characteristics to the viscoelastic behavior of the skin. The goal of this study was to provide both a theoretical and empirical framework for future studies on the use of low-frequency acousto-optical elastography for potential clinical and biomechanical applications.

\subsection{Theoretical Development}

\subsubsection{Physical model}

Skin, while grossly anisotropic, can be considered to be locally isotropic. Thus, for this model, we assume a locally

1083-3668/2004/\$15.00 @ 2004 SPIE 


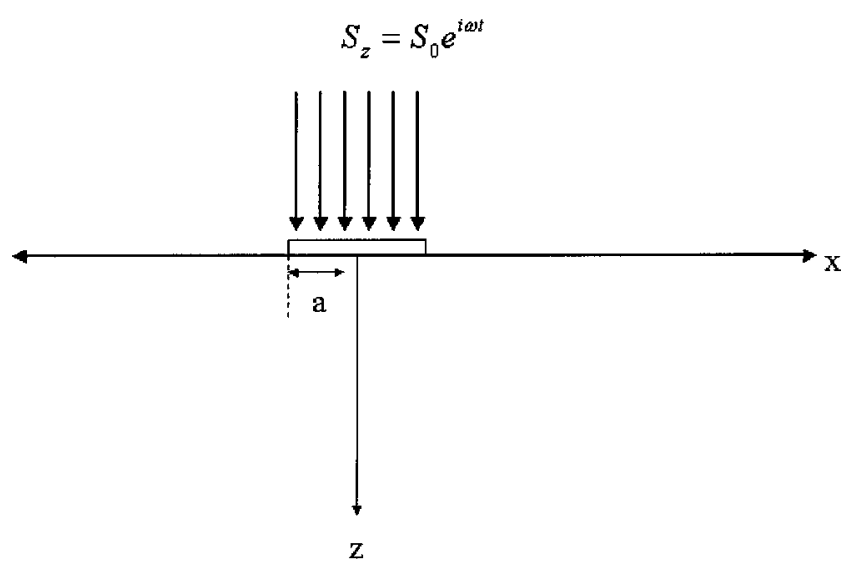

Fig. 1 Isotropic, viscoelastic half-space subjected to a sinusoidal loading over an area of radius $a$.

isotropic half-space subjected to a harmonic external normal force $S_{z}=S_{0} \exp (i \omega t)$ over an area of radius $a$ (Fig. 1). The time-dependent mechanical behavior of the solid material that fills the half-space can be described adequately by the constitutive relation

$$
\sigma_{i j}(t)=\int_{0}^{t} \lambda(t-\tau) \delta_{i j} \frac{d \varepsilon_{k k}}{d \tau} d \tau+\int_{0}^{t} 2 \mu(t-\tau) \frac{d \varepsilon_{i j}}{d \tau} d \tau
$$

where $\sigma_{i j}$ is stress, $\lambda$ and $\mu$ are the two independent Lamé constants, $\delta_{i j}$ is the Kronecker delta $\left(\delta_{i j}=1\right.$ if $i=j ; \delta_{i j}=0$ if $i \neq j), \varepsilon_{k k}=\varepsilon_{11}+\varepsilon_{22}+\varepsilon_{33}$ is strain, and $\tau$ is the time variable of integration. Note that the two viscoelastic functions may not have the same time dependence. Alternatively, engineering functions may be considered instead of the tensorial ones:

$$
\begin{gathered}
G=\mu, \\
E=\frac{G(3 \lambda+2 G)}{(\lambda+G)}, \\
\nu=\frac{\lambda}{2(\lambda+G)}, \\
B=2 G \frac{1+\nu}{3(1-2 \nu)},
\end{gathered}
$$

where $G$ is the shear modulus, $E$ is the elastic modulus, $\nu$ is the Poisson's ratio, and $B$ is the bulk modulus.

Following Zhang et al. ${ }^{5}$ and letting $\mu=\mu_{1}+(\partial / \partial t) \mu_{2}$ and $\lambda=\lambda_{1}+(\partial / \partial t) \lambda_{2}$ where subscript 1 denotes the elastic component and subscript 2 denotes the viscous component, then for the harmonic excitation at angular frequency $\omega$, we have

$$
\begin{aligned}
& \lambda=\lambda_{1}+i \omega \lambda_{2}, \\
& \mu=\mu_{1}+i \omega \mu_{2} .
\end{aligned}
$$

Thus, from Eqs. (1), (2), and (3), the material is viscoelastic in nature, and the harmonic excitation will result in a nonzero imaginary part of $E$. Recalling that the half-space is subjected to a harmonically varying force $S_{z}$, the stress on the material varies sinsuoidally in time as

$$
\sigma(t)=\sigma_{o} \exp (i \omega t) .
$$

The strain will also be sinusoidal, but will lag behind the stress by a phase, or loss, angle $\delta$ :

$$
\varepsilon(t)=\varepsilon_{o} \exp (i \omega t-\delta) .
$$

The phase angle is related to the time lag between the sinusoids by

$$
\delta=\frac{2 \pi \Delta t}{T}
$$

where $T$ is the period of the waveforms. As a result of this phase lag, the complex dynamic modulus $E^{*}$ is represented as a complex number:

$$
E^{*}=\frac{\sigma^{*}}{\varepsilon_{o}^{*}}=\operatorname{Re}(E)+\operatorname{Im}(E) i
$$

The first term on the right-hand side (RHS) of Eq. (7) is the in-phase component and is known as the storage modulus, while the second term on the RHS is the out-of-phase component and is commonly referred to as the loss modulus. The phase angle is a dimensionless measure of the viscoelastic damping of the material and represents the amount of energy lost (e.g., dissipated as heat) during each loading-unloading cycle. The ratio $\operatorname{Im}(E) / \operatorname{Re}(E)$ is commonly referred to as the loss tangent or loss factor and is related to $\delta$ by the equation

$$
\frac{\operatorname{Im}(E)}{\operatorname{Re}(E)}=\omega \tau_{r}=\tan \delta,
$$

where $\tau_{r}$ is the relaxation time of the material and is the ratio of the viscous-to-elastic components of the overall behavior of the material.

In the present scenario, the existence of $\delta$ should manifest as: 1. a decay in the amplitude of the propagating surface wave along $\vec{x}$, and 2 . a significant lag between the imposed acoustic stress wave and the resulting speckle shift. The two manifestations are not unrelated to one another. To see this, it is of interest to relate $\tan \delta$ with the characteristic decay distance, or relaxation distance $x_{\delta}$, which is the propagation distance in which the magnitude of the propagating surface wave (which in the current case is measured by the magnitude of the laser speckle shift), decays to $1 / e$ of its original value, $A_{0}$. The relaxation distance is given by

$$
A=A_{0} \exp \left(-x / x_{\delta}\right) .
$$

Recalling Stokes' relaxation time formulation for viscosity, we can redefine tan $\delta$ in terms of density $\rho_{0}$ and dynamic viscosity $\eta$ as

$$
\tan \delta=\frac{\omega \eta}{\rho_{0} V_{0}^{2}}
$$

where $V_{0}$ is velocity. Without any loss in generality, we can redefine the relaxation time $\tau_{r}$ as 


$$
\tau_{r}=\frac{\eta}{\rho_{0} V_{0}^{2}}
$$

with the denominator of Eq. (11) reflecting the well-known relationship between Young's modulus $E$ density, and wave velocity for an isotropic elastic body $E=\rho V^{2}$. Thus, the viscous relaxation time and $\tan \delta$ can simply be related to each other through Eqs. (8) and (11) as

$$
\frac{\tan \delta}{\omega}=\frac{\eta}{\rho_{0} V_{0}^{2}}=\tau_{r} .
$$

$\tau_{r}$ is simply the time it takes for a wave to travel a distance $x_{\delta}$. Tan $\delta$ and $x_{\delta}$ are then related to each other through $\tau_{r}$. This point is discussed further later on.

\subsubsection{Surface acoustic (Rayleigh) waves}

Consider again the situation of Fig. 1. The harmonic excitation launches a wave polarized in the $x z$ (sagittal) plane with surface normal along $\vec{z}$ and propagating in the $x$ direction. If a plane-strain situation is assumed, then the displacement and velocity components are in $x$ and $z$ directions and there is no coupling to the transverse waves with displacement along $y$ [i.e., shear horizontal (SH) mode], perpendicular to the sagittal plane, so there is no mode conversion or reflection. Assume that the half-space is a semi-infinite solid with a free surface, such that the boundary conditions are that the tangential and normal stresses are zero on the surface at $z=0$.

Because the imposed displacements and therefore strains are small, we adopt the Lagrangian definition of stress and can express the general form of the displacements and the stress components, respectively, as

$$
\begin{gathered}
u_{x}=\frac{\partial \phi}{\partial x}-\frac{\partial \psi}{\partial z}, \\
u_{z}=\frac{\partial \phi}{\partial z}+\frac{\partial \psi}{\partial r}, \\
\sigma_{z z}=\lambda\left(\frac{\partial^{2} \phi}{\partial x^{2}}+\frac{\partial^{2} \phi}{\partial z^{2}}\right)+2 \mu\left(\frac{\partial^{2} \phi}{\partial x^{2}}-\frac{\partial^{2} \psi}{\partial x \partial z}\right), \\
\sigma_{x z}=\mu\left(\frac{\partial^{2} \phi}{\partial x^{2}}+2 \frac{\partial^{2} \phi}{\partial x \partial z}-\frac{\partial^{2} \psi}{\partial x^{2}}\right),
\end{gathered}
$$

where $\phi$ and $\psi$ are potentials for the longitudinal $z$ and transverse $x$ directions, respectively, and $\lambda$ and $\mu$ are the first and second Lamé constants. The origin of Eq. (13) can be seen by defining a scalar and vector potential for the plane-strain situation described earlier:

$$
\vec{u}=\overrightarrow{\nabla \phi}+\vec{\nabla} \times \vec{\psi}
$$

The potentials satisfy the wave equations

$$
\nabla^{2} \phi=\frac{1}{c_{L}^{2}} \frac{\partial^{2} \phi}{\partial t^{2}},
$$

$$
\nabla^{2} \vec{\psi}=\frac{1}{c_{T}^{2}} \frac{\partial^{2} \psi}{\partial t^{2}},
$$

for the longitudinal and shear waves, respectively, with the corresponding wave speeds, $c_{L}$ and $c_{T} .{ }^{6}$

Since we have assumed a plane-strain case, the only nonzero component of $\vec{\psi}$ is in the $y$ direction. Recalling the harmonic time dependence, then the plane wave solutions for $\phi$ and $\psi$ satisfying Eq. (15) are given by

$$
\begin{gathered}
\phi=A_{1} \exp \left[j\left(\omega t-\beta x-\gamma_{L} z\right)\right], \\
\psi=-j A_{2} \exp \left[j\left(\omega t-\beta x-\gamma_{T} z\right)\right],
\end{gathered}
$$

where $A_{1}$ and $A_{2}$ are arbitrary constants and the wave numbers are defined by

$$
\begin{gathered}
\gamma_{L}^{2}=\beta^{2}-k_{L}^{2}, \\
\gamma_{T}^{2}=\beta^{2}-k_{T}^{2} .
\end{gathered}
$$

Here $k_{L}$ and $k_{T}$ are the bulk wave numbers and $\beta=k_{R}$ is the wave number of the harmonic wave traveling along the $x$ axis. $^{7}$

Noting that in the plane-strain case, the normal stresses vanish at the surface and thus $\left.\sigma_{z z}\right|_{z=0}=\left.\sigma_{x z}\right|_{z=0}=0$. Setting the determinant of the coefficients $A_{1}$ and $A_{2}$ equal to zero, then the characteristic Rayleigh equation may be obtained: ${ }^{7}$

$$
\left(\beta^{2}-\gamma_{T}^{2}\right)+4 \beta^{2} \gamma_{L} \gamma_{T}=0 .
$$

Rewriting Eq. (18) in polynomial form with the definitions ${ }^{7}$

$$
\begin{gathered}
\eta \equiv \frac{k_{T}}{\beta}=\frac{c_{R}}{c_{T}}, \\
\xi=\frac{k_{L}}{k_{T}}=\frac{c_{T}}{c_{L}},
\end{gathered}
$$

we arrive at

$$
\eta^{6}-8 \eta^{4}+8\left(3-2 \xi^{2}\right) \eta^{2}-16\left(1-\xi^{2}\right)=0 .
$$

Depending on Poisson's ratio $\nu$ of the material as defined in Eq. (2), several roots to Eq. (20) arise. One of them, the real root with the smallest magnitude, corresponds to the existence of a Rayleigh surface wave. An approximation to this root is given using Bergmann's formula ${ }^{7}$

$$
\eta_{r}=\frac{0.87+1.12 \nu}{1+\nu}
$$

Thus, over the allowable ranges of $0<\nu<0.5$, the Rayleigh wave velocity varies from $0.87 c_{T} \leqslant c_{R} \leqslant 0.96 c_{T}$.

Using the definitions of Eq. (17), the characteristic equation, Eq. (18) may be rewritten in terms of wave speeds as: ${ }^{8}$

$$
r-4 q s=0
$$

where 


$$
\begin{gathered}
q=\left[1-\left(\frac{c_{R}}{c_{L}}\right)^{2}\right]^{1 / 2}, \\
s=\left[1-\left(\frac{c_{R}}{c_{T}}\right)^{2}\right]^{1 / 2}, \\
r=2-\left(\frac{c_{R}}{c_{T}}\right)^{2} .
\end{gathered}
$$

In this fashion, solutions for the particle displacements maybe obtained in an efficient form: ${ }^{8}$

$$
\begin{aligned}
& u_{z}=[A \exp (-q z)-2 s q \exp (-s z)] \cos \beta\left(x-c_{R} t\right), \\
& u_{x}=A q[r \exp (-q z)-2 \exp (-s z)] \sin \beta\left(x-c_{R} t\right),
\end{aligned}
$$

where $A$ is the amplitude, $\beta=2 \pi / \lambda_{R}$ is the Rayleigh wave number, and $\lambda_{R}$ is the Rayleigh wavelength. The Rayleigh wavelength is given by the standard equation

$$
\lambda_{R}=\frac{c_{R}}{f}, \text { where } f=\frac{\omega}{2 \pi} .
$$

Inspection of Eq. (24) reveals that there is an overall elliptical particle motion in the presence of Rayleigh waves. It is this surface particle motion that we wish to track by observing the translating laser speckle. Also, inspection of the equation reveals that $u_{z}$ and $u_{x}$ decay exponentially with $z$, and that since there is no frequency term in Eq. (20), the root $C_{R}$ is nondispersive in an elastic body.

\subsubsection{Estimating speckle shift}

The details of the numerical algorithm used to estimate the speckle pattern shift as a function of acoustic stress imposed by the propagating Rayleigh waves have been given elsewhere, ${ }^{4}$ so only the key features of the algorithm are presented herein.

The crux of the acousto-optical method is to determine the shift $\zeta$ of the speckle pattern as a function of record number (time) and acoustic stress. This shift was calculated using a maximum likelihood approach developed in earlier works. ${ }^{9,10}$ We adopted a frozen speckle model, much like Taylor's frozen turbulence hypothesis, and assume that over a time on the order of a couple sequential exposures of the camera, the structure of the speckle pattern is fixed. The only change with time is its lateral motion. Thus, the speckle motion can be modeled as

$$
g_{j+1}\left(x_{i}\right)=g_{j}\left(x_{i}-\zeta\right),
$$

where the subscript $i$ denotes the pixel (spatial dimension) and the subscript $j$ represents the record (temporal dimension). We assume that the shift $\zeta$ is small compared to a pixel, so that Eq. (26) can be approximated as

$$
g_{j+1}\left(x_{i}\right) \approx g_{j}\left(x_{i}\right)-\zeta g_{j}^{\prime}\left(x_{i}\right) .
$$

This is simply the first two terms of the Taylor series expansion for $g$. To introduce a degree of symmetry into the problem, we inspect the two speckle records on either side of the record of interest,

$$
\left[g_{j+1}\left(x_{i}\right), g_{j-1}\left(x_{i}\right)\right] .
$$

We then determine the $\zeta$ that minimizes the error,

$$
e_{j}^{2}=\sum_{i=1}^{N}\left[g_{j+1}\left(x_{i}+\zeta\right)-g_{j-1}\left(x_{i}-\zeta\right)\right]^{2},
$$

where the summation is over all pixels in the two records. We are thus seeking the $\zeta$ that brings these two records into registration. If we make use of the approximation in Eq. (27), then differentiating with respect to $\zeta$ and rearranging yields the formula

$$
\hat{\zeta}_{j}=\frac{-\sum_{i=1}^{N}\left[g_{j+1}\left(x_{i}\right)-g_{j-1}\left(x_{i}\right)\right]\left[g_{j+1}^{\prime}\left(x_{i}\right)+g_{j-1}^{\prime}\left(x_{i}\right)\right]}{\sum_{i=1}^{N}\left[g_{j+1}^{\prime}\left(x_{i}\right)+g_{j-1}^{\prime}\left(x_{i}\right)\right]^{2}} .
$$

The term in the first square bracket in the numerator is proportional to the first central difference approximation to the derivative;

$$
\frac{\partial g_{j}\left(x_{i}\right)}{\partial t_{j}} \approx \frac{g_{j+1}\left(x_{i}\right)-g_{j-1}\left(x_{i}\right)}{2} .
$$

The spatial derivatives may be approximated similarly:

$$
\begin{gathered}
\frac{\partial g_{j+1}}{\partial x_{i}} \approx \frac{g_{j+1}\left(x_{i+1}\right)-g_{j+1}\left(x_{i-1}\right)}{2}, \\
\frac{\partial g_{j-1}}{\partial x_{i}} \approx \frac{g_{j-1}\left(x_{i+1}\right)-g_{j-1}\left(x_{i-1}\right)}{2} .
\end{gathered}
$$

Note that the speckle shift parameter $\zeta$ is the time rate at which the speckle pattern shifts. The units are pixels/record. This estimator was derived in terms of the shift that minimizes the mean-square error (MMSE) between adjacent records. It is also the maximum likelihood (ML) estimate of the shift. ${ }^{9,10}$

\section{Methods and Materials}

The fundamental method employed in this study was to use an acoustic speaker to supply a low-frequency, sinusoidally varying acoustic stress to the skin. Simultaneously, specific points on the skin were illuminated with coherent laser light and the motions of the backscattered speckle patterns as a function of the driving acoustic stress wave were recorded. The magnitude and the time-resolved pattern of the speckle shift were calculated using the maximum likelihood estimator described before.

All of the experiments were performed using samples of fresh porcine flesh obtained from a local abattoir. The tissue samples consisted of skin, adipose tissue, and underlying muscle tissue. Typical dimensions of the tissue sample were $20 \times 10 \times 5 \mathrm{~cm}$ in the $x, y$, and $z$ dimensions, respectively. Tissues were kept refrigerated until approximately $\frac{1}{2} \mathrm{~h}$ before testing, at which time they were removed from refrigeration, surrounded on all sides, except for the skin surface, with damp paper towels, and allowed to equilibrate to room temperature. To apply the acoustic stress wave, a small speaker $(87 \mathrm{~W}, 7.0 \mathrm{~cm}$ diam) was placed in direct contact with the skin at one end of the sample. The speaker was sinusoidally driven 


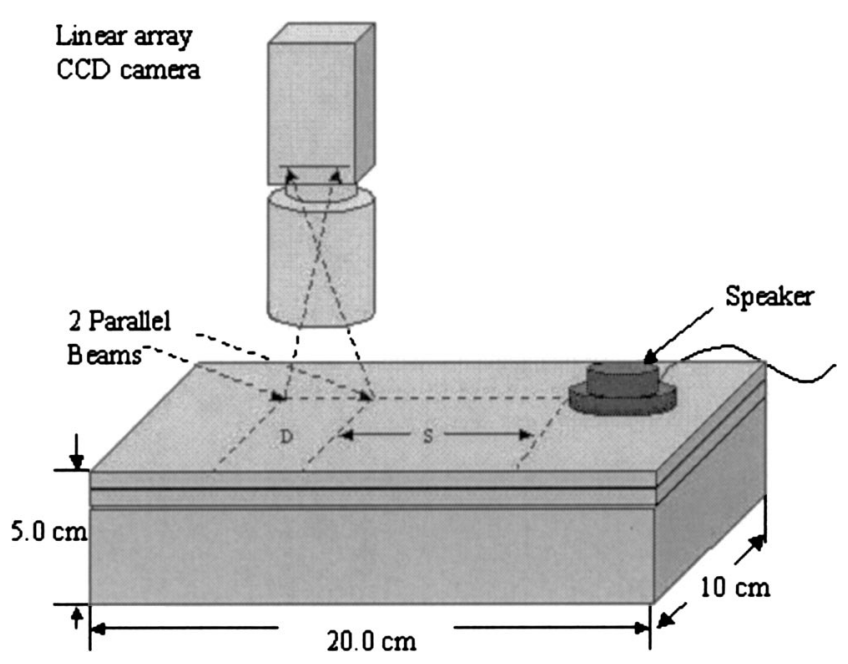

Fig. 2 Acousto-optical arrangement for determining the Rayleigh wavelength and velocity.

at $1 \mathrm{~Hz}$ by a sine wave originating from a function generator. In addition, the speaker was assumed to have remained in solid contact with the skin during excitation. Optical illumination was supplied by a $5-\mathrm{mW}$ green $\mathrm{HeNe}$ laser $(\lambda$ $=543 \mathrm{~nm}$ ) coupled through single-mode fibers. This wavelength was chosen because it restricts our interrogation depth to depths on the order of $100 \mu \mathrm{m}$ or so, thus the measurements are primarily surface measurements.

The backscattered speckle pattern was imaged with a linear array charge-coupled device (CCD) camera that consisted of 5000 pixels with a $7-\mu \mathrm{m}$ pitch. To reduce the amount of data acquired, only the middle 512 pixels were used. A 1:1 telecentric lens was mounted on the camera. The camera was triggered at $50 \mathrm{~Hz}$. Each 1-D exposure of the camera was sequentially arranged into a 2-D array so that exposure number, or time, was along the vertical axis, and pixel number, or space, was along the horizontal axis. We have previously referred to such arrays as stacked-speckle histories. ${ }^{9,10,11}$

\subsection{Rayleigh Wave Velocity and Wavelength}

A standard two-beam configuration was employed to determine the velocity and wavelength of the $1-\mathrm{Hz}$ surface acoustic waves (Fig. 2). The speaker was placed at one end of the skin samples and the HeNe laser was used to illuminate two spots on the skin of approximately $1 \mathrm{~mm}$ diam, so that the laser spots and the speaker formed a straight line along the long axis of the skin. The distance of the spot closer to the speaker was chosen rather arbitrarily at $2.5 \mathrm{~cm}$. This distance was chosen with the awareness that at distances too close to the speaker, the skin is overdriven and the speckles show substantial decorrelation during the acoustic loading-unloading cycle, and at too great of distances, the magnitude of the speckle shift is restrictively small. The distance between the two laser spots varied between approximately 1.4 and $2.0 \mathrm{~cm}$. The purpose of this was to ensure that regardless of the distance between the two spots, the calculated velocity and wavelength did not vary. The linear array CCD camera was aligned with the array parallel to the line formed by the speaker and the illuminated spots. A slight misfocus of $1 \mathrm{~cm}$ was purposely induced to ensure that the speckles translated in space as opposed to simply boiling, as would be the case for a perfectly focused system. ${ }^{12}$

The Rayleigh wave velocity $C_{R}$ was determined by the equation

$$
C_{R}=\frac{\omega D}{\phi_{1}-\phi_{2}},
$$

where $\omega$ is the angular frequency of the driving waveform, $D$ is the distance between the two laser spots, and $\phi_{1}-\phi_{2}$ is the difference between the phases of the sinusoids that describe the motion in the stacked speckle histories generated from each of the illuminated spots [Eqs. (26) through (32)]. Once the velocity $C_{R}$ was determined, the Rayleigh wavelength $\lambda_{R}$ could then be estimated from Eq. (25).

Finally, once both $C_{R}$ and $\lambda_{R}$ were determined, the relative particle motions in $\vec{x}$ and $\vec{z}$ were estimated using Eq. (24).

This procedure was conducted on three different locations each on five separate skin samples.

\subsection{Decay in Amplitude of Surface Acoustic Wave}

The physical model described before predicts an exponential decay in the magnitude of the acoustic wave with distance from the acoustic source [Eq. (9)]. To determine the characteristic decay distance, or relaxation distance $x_{\delta}$, which is the propagation distance in which the magnitude of the propagating surface wave as measured by the magnitude of the speckle shift decays to $1 / e$ of its original value $A_{0}$, an experimental arrangement similar to that described earlier was employed. However, only a single illumination beam was used, and the location of this illuminated spot was moved sequentially further away from the speaker. The magnitude of the speckle shift as a function of distance from the speaker was determined at each point distant from the speaker. As before, the speaker was driven sinusoidally at $1 \mathrm{~Hz}$. Measurements were made at distances ranging from approximately 3.3 to $6.4 \mathrm{~cm}$ from the edge of the speaker. Beyond this latter distance, the shift in the speckle histories became prohibitively small (due to attenuation of the surface wave) to a point where the shift could no longer be analyzed with any degree of confidence.

Stacked speckle histories were generated for each point, and the magnitude of the speckle shift [Eqs. (26) through (32)] was taken to be proportional to the magnitude of the acoustic wave at each distance from the speaker. The magnitude of the speckle shift was plotted against distance, and the data were fit in a least-squares sense to an equation of the form

$$
y=A_{o} \exp \left[\left(-x / x_{\delta}\right)\right]
$$

where $x$ is the distance from the edge of the speaker along $\vec{x}$, and the characteristic decay distance $x_{\delta}$ was solved as a free variable.

This procedure was repeated on 3 separate samples of skin.

\subsection{Tan $\delta$ of Porcine Skin}

While the earlier velocity measurements relied on evaluating the phase difference between oscillating speckle patterns from two illuminated spots, the measurement of $\tan \delta$ relies on determining the relative phase difference between the driving 


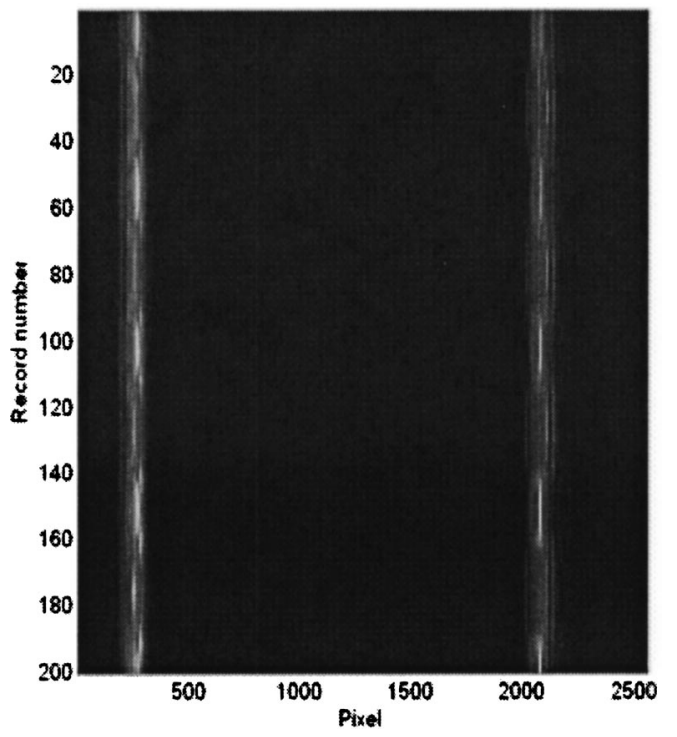

Fig. 3 Dual stacked-speckle history showing the translation of the speckle patterns at two distinct spots on the skin separated by $2.0 \mathrm{~cm}$ due to the sinusoidal acoustic stress wave.

acoustic sinusoid and the sinusoidal shift in the speckle pattern from a single illuminated spot. In this case, the illuminated spot was located $5 \mathrm{~cm}$ from the edge of the speaker.

The speaker was again driven with a $1-\mathrm{Hz}$ sinusoid from a function generator and the backscattered speckle pattern was imaged with the linear array CCD camera. As before, stacked speckle histories were generated and the speckle shift was plotted along with the driving waveform as a function of time (or record number).

Based on the measured Rayleigh wave velocity $C_{R}$, a correction was made for time of flight of the surface acoustic wave and the phase difference $\delta$ between the driving acoustic wave. The resulting speckle shift was calculated. The phase of each waveform was determined as the arctangent of the ratio between the imaginary and real parts of the waves. The tangent of the phase difference was taken to be the desired variable, $\tan \delta$. The phase delay due to the electronics (i.e., the time delay between the time the signal is sent to the speaker and the coil actually moves) was considered to be negligibly small and was ignored in this analysis.

This procedure was conducted on three different locations each on five separate skin samples.

\section{Results}

\subsection{Rayleigh Wave Velocity and Wavelength}

A typical dual-stacked speckle history is shown in Fig. 3. This figure shows the sinusoidal motion of the backscattered speckle patterns from the two illuminated spots on the porcine skin sample. The spots were separated by a distance $D$ $=2.0 \mathrm{~cm}$, and because of this separation, the sinusoids describing the motions are very slightly out of phase with each other (Fig. 4). This phase difference is the denominator of Eq. (33).

Based on Eq. (33), the mean velocity $C_{R}$ of the $1-\mathrm{Hz}$ surface acoustic waves was estimated to be $2.9 \pm 1.8 \mathrm{~m} / \mathrm{s}$. No

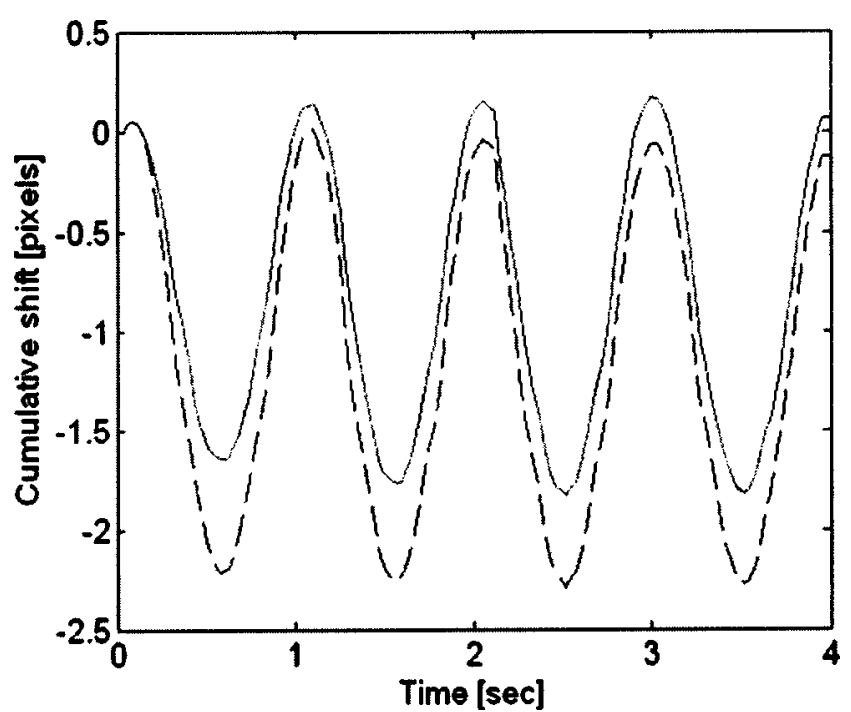

Fig. 4 Cumulative speckle shift as a function of time for each of the speckle histories shown in Fig. 3. The two waves are very slightly out of phase with each other. For the dashed line, the peak shift of -2.25 pixels in the image plane corresponds to a peak shift of 0.16 $\mu \mathrm{m}$ of the skin surface.

difference (student's $t$-test, $p>0.05$ ) in $C_{R}$ was found, regardless of the separation distance between the two spots, lending a degree of confidence to the measurements. Figure 5 shows the mean and standard deviation of the velocity measurements at each of the four separations investigated. Using the value $C_{R}=2.9 \pm 1.8 \mathrm{~m} / \mathrm{s}$, the Rayleigh wavelength $\lambda_{R}$ was then calculated using Eq. (25). Based on this equation, $\lambda_{R}$ $=2.9 \pm 1.8 \mathrm{~m}$ for a $1-\mathrm{Hz}$ surface wave. This long wavelength indicates that our measurements are all in the acoustic nearfield.

With knowledge of $C_{R}$ and $\lambda_{R}$, the relative particle displacements in $u_{x}$ and $u_{z}$ as a function of depth can be estimated via Eq. (24), allowing $\nu=0.45$ (a reasonable number for Poisson's ratio of biological tissue) and $C_{L}=1500 \mathrm{~m} / \mathrm{s}$. The results of this theoretical prediction are plotted in Fig. 6 . The primary result here is that both components of particle displacement have decay constants $\sim 1 \lambda_{R}$, indicating that the wave is in fact a surface wave. This depth, however, is substantially greater than the dimensions of skin, indicating that subsurface features may influence the behavior of the wave as observed on the surface. However, it should be appreciated that the actual displacements, even on the surface, are very small, typically submicron, and the attenuation of the wave is rapid. For example, from Fig. 5, which plots the cumulative speckle shift in the image plane for two illuminated spots located 2.5 and $4.5 \mathrm{~cm}$, respectively, from the speaker, we can approximate the motion on the skin (object plane). For the spot located closest to the speaker $(2.5 \mathrm{~cm}$ away, dashed line in Fig. 5), a peak shift of $\sim 2.25$ pixels was observed for this specific experiment. For a camera pitch of $7 \mu \mathrm{m}$, this corresponds to a shift of $15.8 \mu \mathrm{m}$ in the image plane. Since a $1: 1$ telecentric lens was used with a misfocus distance of $1.0 \mathrm{~cm}$, this motion relates to a peak-to-peak shift in the object plane (i.e., on the skin) of only $0.16 \mu \mathrm{m}$. This peak shift was over $\frac{1}{2}$ of an acoustic cycle. Since the sample rate was $50 \mathrm{~Hz}$, the 


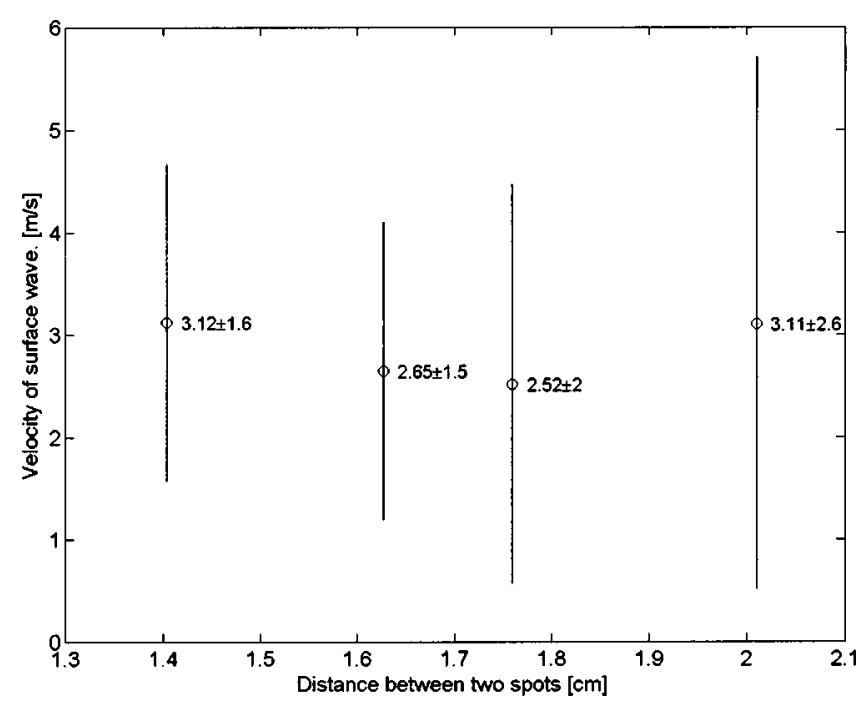

Fig. 5 Velocity and standard deviation of the surface wave at four different separation distances between the two laser spots. There was no significant difference between the different separation distances in the measured velocity. Velocities are in $\mathrm{m} / \mathrm{s}$. The overall mean velocity $C_{R}$ was $2.9 \pm 1.8 \mathrm{~m} / \mathrm{s}$.

$0.16-\mu \mathrm{m}$ shift occurred over 25 samples, or $0.5 \mathrm{~s}$, yielding a shift of $\sim 6.4 \mathrm{~nm}$ per record.

\subsection{Decay in Amplitude of Surface Acoustic Wave}

The relative amplitude of the surface acoustic wave, as measured by the magnitude of the speckle motion, decayed exponentially as a function of distance from the acoustic source. The results are plotted in Fig. 7. The line shows the fit to Eq. (34), and the characteristic decay distance $x_{\delta} \cong 2.9 \mathrm{~cm}$. The equation describing the relative decay of the amplitude was

$$
y=\exp [(-x / 2.88)]
$$

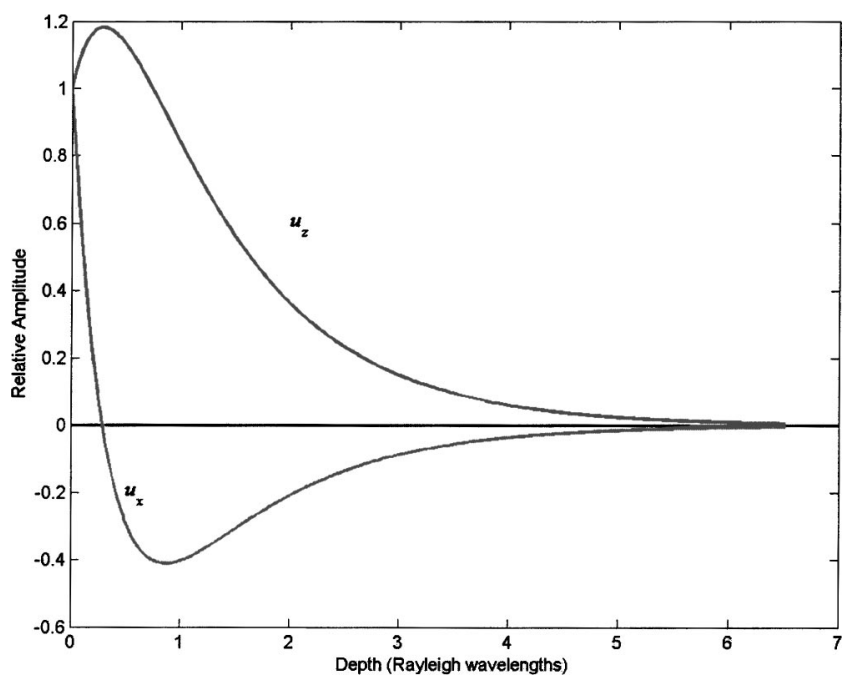

Fig. 6 Relative amplitudes of the $x$ and $z$ components of displacement of the skin Rayleigh wave as a function of depth based on the theoretical Eq. (24). The Poisson's ratio was assumed to be 0.45 and $C_{L}$ was assumed to equal $1500 \mathrm{~m} / \mathrm{s}$.

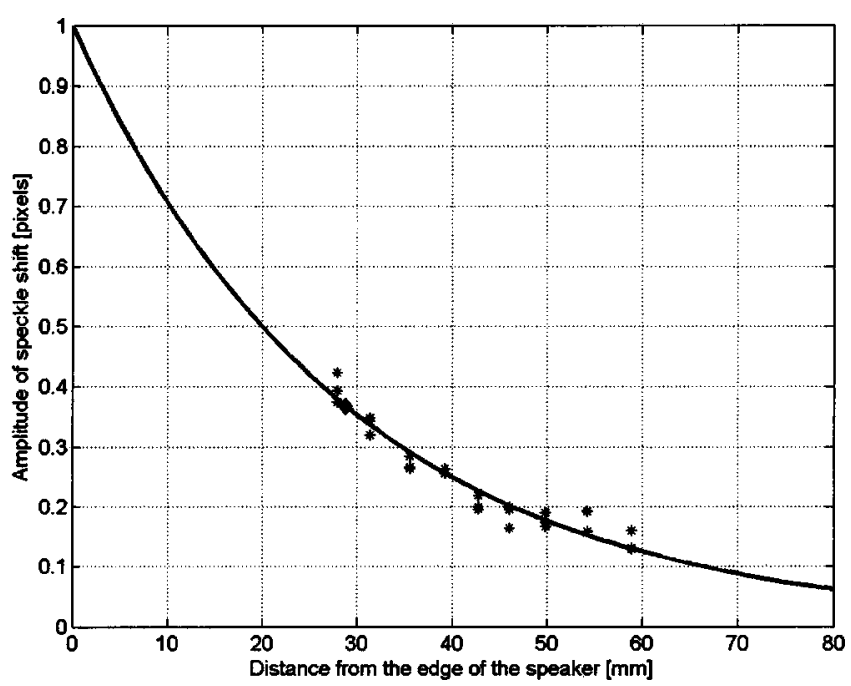

Fig. 7 Relative decay in speckle shift as a function of distance from the speaker. The decay followed a negative exponential with $x_{\delta}$ $\cong 2.9 \mathrm{~cm}$, as indicated by the solid diamond.

where $y$ is the peak-to-peak magnitude of the speckle shift and the decay constant is in centimeters.

\subsection{Tan $\delta$ of Porcine Skin}

Based on the phase differences between the driving acoustic sinusoid and the sinusoid describing the time-resolved speckle shift, the mean value of $\tan \delta$ was $0.14 \pm 0.07$, or a phase angle of approximately $8 \mathrm{deg}$. Table 1 shows the actual values for each skin sample and their standard deviations. A phase angle of $8 \mathrm{deg}$ physically means that the strain in the skin sample lagged behind the imposed acoustic stress by $8 \mathrm{deg}$ when the skin was subjected to a dynamic loading regime at 1 $\mathrm{Hz}$.

\section{Discussion}

The slow velocity $C_{R}$ of the surface waves and corresponding long wavelengths measured in this study are consistent with the surface wave velocities measured in previous studies by other authors using very different methods. ${ }^{1-3}$ Thus, it is likely that the actual velocity of $1-\mathrm{Hz}$ surface acoustic waves in skin is on the order of $3.0 \mathrm{~m} / \mathrm{s}$, and that the Rayleigh wave-

Table 1 Measured $\tan \delta$ values for five skin samples. Each value is the mean of three measurements.

\begin{tabular}{cc}
\hline Skin sample & Tan $\delta$ (mean \pm s.d.) \\
\hline 1 & $0.166 \pm 0.04$ \\
2 & $0.237 \pm 0.12$ \\
3 & $0.154 \pm 0.07$ \\
4 & $0.140 \pm 0.04$ \\
5 & $0.057 \pm 0.03$ \\
Overall & $0.14 \pm 0.07$ \\
\hline
\end{tabular}


length is of the same order. Vexler, Polyansky, and Gorodetsky $^{13}$ examined the propagation velocity of shear waves in human skin at an acoustic frequency of $5.6 \mathrm{KHz}$ and found that the velocities ranged between approximately 20 and $80 \mathrm{~m} / \mathrm{s}$, depending on the direction of wave propagation in relation to the anisotropic behavior of the skin. They used these velocities as indirect measurements of the viscoelasticity of the skin for determining the physiological status of skin.

As another confirmation of the value of $C_{R}$, Eq. (12) can be used to solve for $\tau_{r}$. Using Eq. (12), and allowing tan $\delta=0.14$, the viscous relaxation time $\tau_{r}=0.02 \mathrm{~s}$. Physically, this can be interpreted that it takes $0.02 \mathrm{~s}$ for the surface acoustic wave to travel the distance $x_{d}=2.9 \mathrm{~cm}$. Using these values to solving for velocity $C_{R}=1.45 \mathrm{~m} / \mathrm{s}$, which is lower than the directly measured velocity of $2.9 \pm 1.8 \mathrm{~m} / \mathrm{s}$, but still within the standard deviation of the measurements. A possible explanation for this discrepancy may be that the acoustic field is probably not quite flat, but is probably very slightly expanding. Recall that the diameter of the speaker was only 3 $\mathrm{cm}$ less than the width of the tissue samples. Thus, the measured decay in the relative amplitude of the acoustic stress wave with distance from the speaker is the result of some combination of wavefront expansion and the viscous nature of the tissue. If the wavefront was truly flat, the measured and calculated values of $C_{R}$ would likely be even closer.

The decay in the amplitude of the acoustic wave with distance from the speaker is indicative of the viscous nature of the tissue. Although not investigated here, the characteristic decay constant should change with a change in frequency, as indicated by the description of the physical model from before. Furthermore, it is very likely that this decay constant will change with tissue conditions as well, including location of the skin sample on the body, hydration levels, age and physiological (i.e., healthy or diseased) state. These are certainly issues that need to be investigated.

The variation in $\tan \delta$ values between samples is consistent with the variation seen between individuals in Karzakov and Klochkov's ${ }^{2}$ study on the low-frequency mechanical properties of skin tissues in the human arm. Potts, Chrisman, and Buras $^{1}$ also noted large variations between individuals. This variation simply reflects the natural variation in tissues, and the fact that the mechanical properties of skin are very dependent on location on the body and physiological parameters. It has been shown, however, that the values of $\tan \delta$ of skin can be varied in a consistent fashion through the local injection of cross-linking agents (decreases $\tan \delta$ ) or enzymatic agents to partially digest the tissue (increases $\tan \delta$ ). ${ }^{14}$

The long wavelength of the Rayleigh wave $(\sim 3 \mathrm{~m})$ brings into question the assertions that the skin sample can be modeled as a semi-infinite medium, and that the method evaluates the viscoelastic properties of the skin only, and not the entire tissue slab, including fat and muscle tissue. However, careful consideration of the results and of the optical sensing of the acoustic wave supports both assertions. First, the rapid attenuation of the surface wave as demonstrated by the short decay constant in the $x$ and $z$ directions by Fig. 6, along with the very small surface motions observed (see Results in Sec. 3), leads to the observation that the surface wave has attenuated dramatically before reaching a boundary, and reflections from boundaries are not a significant factor. Indeed, the smooth sinusoidal motion of the speckle patterns (Figs. 3 and 4) sup- ports this observation. Reflections of the acoustic wave back into the measurement area would lead to period speckle decorrelation and nonsinusoidal shifts in the pattern of the speckle shift. Neither of these phenomena was observed. Thus, there is no reason to model the tissue slab as a waveguide, and it is better modeled as a semi-infinite medium, as was done here. Second, making the reasonable assumption that porcine skin has similar optical properties to human skin, ${ }^{15}$ then the approximate roundtrip probing depth of the 543-nm HeNe laser was on the order of $150 \mu \mathrm{m}$. Thus, the measurements of the viscoelastic behavior of the tissue were limited to about that depth, and not the entire thickness of the tissue slab.

Clearly, the variation in the measured mechanical properties of skin makes it somewhat impractical to rely solely on absolute values of the mechanical properties for diagnostic purposes. However, it seems reasonable that relative hydration levels of the skin could be determined in this fashion. On the other hand, variations in the local tissue mechanical properties may provide a means to interrogate the tissue. For example, if a large area of skin, including a suspected pathological site, were to be illuminated and a sequence of speckle images were taken as the skin is acoustically stressed, then neighborhood variations in the speckle shift across the images could be determined by extending the speckle shift algorithm to three dimensions (the third dimension being time). ${ }^{16}$ In this manner, the tissue surrounding the suspect tissue would serve as an internal control. Stiffer tissue areas would exhibit less speckle motion, while less stiff areas would exhibit greater motion under the same acoustic stress, indicating local variations in mechanical properties.

\section{Acknowledgments}

This grant was funded in part by NSF grant numbers BES0196172 and BES-0201841, and NIH grant number 5R24EB000224-04. The authors wish to thank Deborah Baker and Jon Adams for their assistance in making some of the tan $\delta$ measurements.

\section{References}

1. R.O. Potts, D.A. Chrisman, and E.M. Buras, "The dynamic mechanical properties of human skin in vivo," J. Biomech. 16(6), 365-372 (1983).

2. V.V. Kazakov and B.N. Klochkov, "Low frequency mechanical properties of the soft tissue of the human arm," Biophysics (Engl. Transl.) 34(4), 742-747 (1989).

3. S. Ganesan, C. Man, and S.J. Lai-Fook, "Generation and detection of lung stress waves from the chest surface," Respir. Physiol. 110, 19-32 (1997)

4. S.J. Kirkpatrick, M.T. Hinds, and D.D. Duncan, "Acousto-optical characterization of the viscoelastic nature of a nuchal elastin tissue scaffold," Tissue Eng. 9(4), 645-656 (2003).

5. X. Zhang, T.J. Royston, H.A. Mansy, and R.H. Sandler, "Radiation impedance of a finite circular piston on a viscoelastic half-space with application to medical diagnostics," J. Acoust. Soc. Am. 109(2), 795802 (2001).

6. C.T. Schröder and W.R. Scott, Jr., "On the complex conjugate roots of the Rayleigh equation: The leaky surface wave," J. Acoust. Soc. Am. 110(6), 2867-2877 (2001).

7. J.D.N. Cheeke, Fundamentals and Applications of Ultrasonic Waves, CRC Press, Boca Raton, FL (2002).

8. K. Worden, "Rayleigh and Lamb waves-basic principles," J. Strain Anal. 37, 167-172 (2001).

9. D.D. Duncan and S.J. Kirkpatrick, "Processing algorithms for tracking speckle shifts in optical elastography of biological tissues," $J$. Biomed. Opt. 6(4), 418-426 (2001). 
10. D.D. Duncan and S.J. Kirkpatrick, "Performance analysis of a maximum-likelihood speckle motion estimator," Opt. Express 10, 927-941 (2002).

11. D.D. Duncan, S.J. Kirkpatrick, F.F. Mark, and L.W. Hunter, "Transform method of processing for speckle strain rate measurements," Appl. Opt. 33, 5177-5186 (1994).

12. J.W. Goodman, "Statistical properties of laser speckle patterns," in Laser Speckle and Related Phenomena, J.C. Dainty, ed., SpringerVerlag, Berlin (1984).

13. A. Vexler, I. Polyansky, and R. Gorodetsky, "Evaluation of skin vis- coelasticity and anisotropy by measurement of speed of shear wave propagation with viscoelasticity skin analyzer," J. Invest. Dermatol. 113, 732-739 (1999).

14. S.J. Kirkpatrick and D.D. Duncan, "Acosuto-optical assessment of skin viscoelasticity," Proc. SPIE 4961, 209-220 (2003).

15. R.R. Anderson and J.A. Parrish, "The optics of human skin," J. Invest. Dermatol. 77, 13-19 (1981).

16. D.D. Duncan and S.J. Kirkpatrick, "Maximum-likelihood estimators for one and two-dimensional speckle motion," Proc. SPIE 4961, 202-208 (2003). 